\title{
Tiotropium improves walking endurance in COPD
}

\author{
M-E. Bédard*, C. Brouillard*, V. Pepin\#, S. Provencher*, J. Milot*, Y. Lacasse*, \\ P. Leblanc* and F. Maltais*
}

ABSTRACT: The primary objective of this study was to evaluate the effects of a 3-week treatment with tiotropium on walking capacity in chronic obstructive pulmonary disease (COPD).

After familiarisation with study procedures, 36 patients were randomised to receive tiotropium $18 \mu \mathrm{g}$ once daily or a matching placebo in a double-blind, parallel-group study. Pre- (trough) and 2-h post-dose pulmonary function was measured. An endurance shuttle walk was then completed. The same procedures were repeated after 3 weeks of treatment. Ventilatory parameters were monitored during exercise.

At 3 weeks, tiotropium significantly improved walking endurance time in comparison with placebo, with a mean $\pm S D$ between-group difference of $128 \pm 141 \mathrm{~s}(\mathrm{p}=\mathbf{0 . 0 1 7})$. At 3 weeks, trough values for forced expiratory volume in $1 \mathrm{~s}(\mathrm{FEV} 1)$ and forced vital capacity (FVC) were significantly improved with tiotropium in comparison with placebo. The post-dose response to tiotropium was statistically superior to placebo after the first dose and after 3 weeks of treatment for FEV 1 , FVC and inspiratory capacity. Ventilation and tidal volume at the end of walking were significantly improved with tiotropium.

3 weeks of tiotropium resulted in a greater walking endurance in patients with COPD. Improvements in FEV1, maximal ventilation and tidal volume may contribute to this enhanced exercise capacity.

KEYWORDS: Bronchodilation, chronic obstructive pulmonary disease, exercise, shuttle walking test, tiotropium, walking

ong-acting bronchodilators, such as tiotropium, aclidinium, salmeterol and formeterol, are effective in improving exercise tolerance in patients with chronic obstructive pulmonary disease (COPD). In well-designed, randomised, placebocontrolled trials, these bronchodilators have been convincingly shown to improve endurance time, dyspnoea and hyperinflation during constant-load cycling endurance tests in COPD [1-5]. Although the symptomatic and functional benefit associated with these drugs is felt to be clinically relevant, important issues remain to be resolved in this regard. For instance, we do not know whether the observed improvement in cycling capacity translates into better performance in different activities of daily living, such as walking.

We recently documented, in clinical trials, the responsiveness of the endurance shuttle walk test (ESWT) to detect improvement in functional status following the administration of ipratropium bromide [6] and salmeterol [7] in patients with COPD. The ESWT appears as an exercise testing modality that is particularly well suited to evaluating the efficacy of pharmacotherapy in COPD because it is more responsive than the 6-min walking test in this context [8] and may be more relevant to activities of daily living compared with cycling. Based on these observations, we reasoned that tiotropium should also improve walking time during an ESWT in COPD.

The primary objective of this study was to compare, at 3 weeks, the efficacy of tiotropium to improve walking endurance time in comparison with placebo in patients with COPD. Secondary objectives were to assess changes in endurance time after the first dose of study medication, as well as the changes in pulmonary function after the first dose of study medication and at 3 weeks in comparison with baseline. We also assessed the impact of study treatment on the physiological responses to exercise at 3 weeks in comparison with baseline.

\section{MATERIALS AND METHODS}

\section{Study population}

Subjects included in this study were clinically stable patients with COPD, who were aged $\geqslant 50 \mathrm{yrs}$, had a forced expiratory volume in $1 \mathrm{~s}$ (FEV1) $\leqslant 70 \%$ predicted, a FEV1/forced vital capacity (FVC) $<70 \%$

\section{AFFILIATIONS}

${ }^{*}$ Centre de Recherche, Institut Universitaire de Cardiologie et de Pneumologie de Québec, Université Laval, and

${ }^{\text {\#} C e n t r e ~ d e ~ R e c h e r c h e, ~ H o ̂ p i t a l ~ d u ~}$ Sacré-Coeur de Montréal, Concordia University, Québec, Canada.

\section{CORRESPONDENCE}

F. Maltais

Centre de Recherche

Institut Universitaire de Cardiologie et de Pneumologie de Québec

2725

Chemin Ste-Foy

Québec

G1V 4G5 Canada

E-mail: francois.maltais@

med.ulaval.ca

Received:

April 062011

Accepted after revision: May 272011

First published online:

June 232011 
and a smoking history of $\geqslant 10$ pack-yrs. Exclusion criteria were as follows: 1) COPD exacerbation in the last 2 months; 2) history of asthma; and 3) supplemental oxygen therapy or significant oxygen desaturation (arterial oxygen saturation $<85 \%$ ) either at rest or during exercise. Patients with a history of left ventricular dysfunction (left ventricular ejection fraction $<50 \%$ ), a diagnosis of cancer within the past $5 \mathrm{yrs}$, and neuromuscular or locomotive impairment affecting the ability to walk were also excluded. In the case of COPD exacerbation during the study protocol, patients were treated as recommended by the investigator and withdrawn from the study. This was done because patients would not have time to recover fully before the assessment of the primary endpoint in this 3-week study. Patients were not involved in pulmonary rehabilitation at the time of the study. The research protocol was approved by the institutional ethics committee, and a signed, informed consent was obtained from each subject.

\section{Study design}

This single-centre, double-blind, randomised, parallel-group study required five visits to the research facility. All study procedures, including the recruitment of patients, were performed at the Institut Universitaire de Cardiologie et de Pneumologie de Quebec, Quebec, Canada, a tertiary care hospital that is the referral hospital for respiratory diseases in the eastern part of the province of Quebec. The first visit included review of the consent form, pulmonary function testing and one maximal incremental shuttle walk test (ISWT). The following two visits were used to familiarise participants with the ESWT and establish their baseline endurance time. The goal of familiarisation was to reduce the learning effect that typically occurs when an individual completes the same endurance test several times [9]. One additional ESWT could be performed on a subsequent visit in case of nonreproducibility of their endurance time (difference $>2 \min$ or $10 \%$ ) on visits 2 and 3 . If reproducibility was not reached despite this supplementary visit, the patient was excluded. If the endurance time was $>20 \mathrm{~min}$ at the maximal walking speed, the patient was also excluded.

At visit 4, patients were randomised to receive placebo or tiotropium (Spiriva ${ }_{\mathbb{R}}$; Boehringer Ingelheim, Burlington, Ontario, Canada) for 3 weeks at $18 \mu \mathrm{g}$ once daily, in a double-blind fashion using the HandiHaler ${ }^{\circledR}$ device (Boehringer Ingelheim $\mathrm{GmbH}$, Ingelheim, Germany), which was identical in appearance for the placebo and the active medication. Randomisation was performed by the study pharmacist using a randomisation table. The study medication was provided by the pharmacist who ensured concealment of allocation and kept a log of the allocation group for each recruited patient. Pulmonary function testing was performed before (pre-dose) and $120 \pm 20 \mathrm{~min}$ after (post-dose) inhalation of the first study dose. An ESWT was then performed. Patients were provided enough medication for the study duration and discharged. The same procedures were completed 3 weeks later, at visit 5 .

The following medications were allowed throughout the study if used for $\geqslant 4$ weeks before the trial: as-needed short-acting $\beta_{2}$-agonists (stopped $6 \mathrm{~h}$ preceding visits $2-5$ ), long-acting $\beta_{2^{-}}$ agonists (stopped $48 \mathrm{~h}$ preceding visits $2-5$ ), inhaled corticosteroids, and a combination of long-acting $\beta_{2}$-agonists and inhaled corticosteroids (stopped $48 \mathrm{~h}$ preceding visits $2-5$ ). Patients on tiotropium were switched to ipratropium bromide $40 \mu \mathrm{g}$ q.i.d. $\geqslant 4$ weeks prior to the trial. Ipratropium bromide was not taken in the 6-h period preceding visits 2 and 3, and its use was not allowed from $6 \mathrm{~h}$ prior to visit 4 and for the remaining duration of the study. Theophyllines were not allowed.

\section{Outcome variables}

The primary outcome of this study was the change in endurance time at 3 weeks (visit 5) versus baseline (the last familiarisation ESWT). Secondary outcomes included: 1) change in endurance time after the first dose of study medication (visit 4) versus baseline; 2) trough (pre-dose) pulmonary function at 3 weeks (visit 5) in comparison with baseline (pre-dose values at visit 4); 3) changes in pulmonary function after the first dose of study medication (visit 4) and after the last dose of study medication in comparison with baseline; and 4) impact of study treatment on the physiological response to exercise at 3 weeks in comparison with baseline.

\section{Study procedures}

Pulmonary function testing

Standard pulmonary function testing, including spirometry, lung volumes and diffusing capacity of the lung for carbon monoxide $(D \mathrm{~L}, \mathrm{CO})$ were measured at the first visit according to previously described guidelines [10-12]. Results were compared with predicted normal values from the European Community for Coal and Steel/European Respiratory Society (ERS) [13]. Spirometry and lung volume measurements were repeated before and $120 \pm 20$ min after inhalation of study medication at visits 4 and 5 .

\section{ISWT}

Peak walking capacity was determined with the ISWT. As originally described by SINGH et al. [14], the test was performed in an enclosed corridor on a flat 10-m long course. The course was identified by two cones, each positioned $0.50 \mathrm{~m}$ from either end to allow patients to walk in a circle and avoid the need for abrupt changes in direction. Patients walked at a pre-determined rhythm, as dictated by an audio signal emitted at each end of the course. Walking speed was initially set at $0.50 \mathrm{~m} \cdot \mathrm{s}^{-1}$ and then increased by $0.17 \mathrm{~m} \cdot \mathrm{s}^{-1}$ every minute until the patient reached a symptom-limited maximal capacity. Patients received standardised instructions to walk for as long as possible. Encouragement was provided to ensure maximal performance during the test.

\section{ESWT}

Endurance walking capacity was determined with the ESWT. It was performed on the same course as the ISWT in accordance with published guidelines [15]. After $90 \mathrm{~s}$ of warm-up, walking speed was set at a speed corresponding to $80 \%$ of peak oxygen uptake $\left(\mathrm{VO}_{2}\right)$, as predicted from the ISWT. Before each ESWT, patients received standardised instructions to walk for as long as possible, although there was a pre-determined 20-min maximum. As the effects of encouragement on walking performance have been demonstrated [16], no encouragement was given to patients throughout the test. Reproducibility criteria were set at either $\leqslant 2 \mathrm{~min}$ or $10 \%$ between consecutive ESWTs. The final measure was endurance time, expressed in seconds, excluding the 90-s warm-up period. The ESWT was supervised without knowledge of the spirometry results. The proposed minimal important difference for this test is mean 65 s (95\% CI 45-85 s) [17]. 


\section{Physiological measures}

During each exercise test, cardiac and ventilatory parameters (heart rate, arterial oxygen saturation measured by pulse oximetry, respiratory rate, ventilation and gas exchange) were measured and recorded using a portable metabolic system (Jaeger Oxycon Mobile,; Viasys Healthcare GmbH, Hoechberg, Germany). This system, both light and compact, allows breathby-breath measurement of pulmonary gas exchange parameters during exercise. It consists of a face mask, electrocardiogram recorder, pulse oximeter, battery, transmitting unit and receiving unit. The volume sensor and gas analysers were calibrated before each test. The intensity of dyspnoea and perception of leg fatigue were evaluated at rest and at end-exercise using the modified 10point Borg scale [18]. The intensity of dyspnoea was also evaluated with this scale at 2-min intervals during the exercise tests. At the end of each test, patients were asked to identify the main reason for stopping the test.

\section{Statistical analysis}

Results are reported as mean $\pm \mathrm{SD}$, except in the figures where standard errors are used for clarity purposes. The sample size calculation was based on the premise that the improvements in the walking endurance time with tiotropium should be of similar magnitude to that seen with ipratropium bromide in a previous study (164 s) with a similar standard deviation (177 s) [6]. It was calculated that 17 patients in each treatment arm would be necessary for this study to reach a power of 0.80 at $p=0.05$ significance with a two-tailed statistical test. Taking into account a $25 \%$ attrition rate, we planned to recruit 45 patients for this study. Between-group comparisons for baseline characteristics were performed using unpaired t-tests. The Chi-squared test was used for categorical variables. Normality assumption was verified with the Shapiro-Wilk's test. Between-group differences were calculated when appropriate. The effects of treatment on walking endurance time were analysed with a repeated measures ANOVA in which a heterogeneous residual variance model specific to time of measurements was adjusted. The changes in pulmonary function parameters and in physiological variables during exercise were also analysed with a repeated measures ANOVA model. Isotime was defined as the latest exercise time that was reached both at baseline and at 3 weeks (visit 5) during the ESWT. Significance level was set at $\mathrm{p}=0.05$ for all analyses.

\section{RESULTS}

\section{Subjects}

The study flow chart is presented in figure 1.68 patients were screened, and 32 were excluded due to various ineligibility reasons. Thus, 36 patients were randomised in a 1:1 ratio to tiotropium or placebo at visit 4 . One patient in each group failed to complete the protocol because of a COPD exacerbation. Thus, 34 patients constituted the analysis set, 17 in the tiotropium arm and 17 in the placebo arm. Subject characteristics are presented in table $1 . \sim 70 \%$ of participants were male. Patients had, on average, moderate-to-severe airflow obstruction with mild hyperinflation, gas trapping and reduction of $\mathrm{DL}, \mathrm{CO}$ at rest. Peak symptom-limited $V_{2}$ was reduced, averaging only $17 \mathrm{~mL} \cdot \mathrm{kg}^{-1} \cdot \mathrm{min}^{-1}$, and the ventilatory reserve was reduced with a mean minute ventilation/maximum voluntary ventilation ratio $>90 \%$. Baseline endurance time was 390-400 s. Apart from body mass index, which was higher in the tiotropium group in

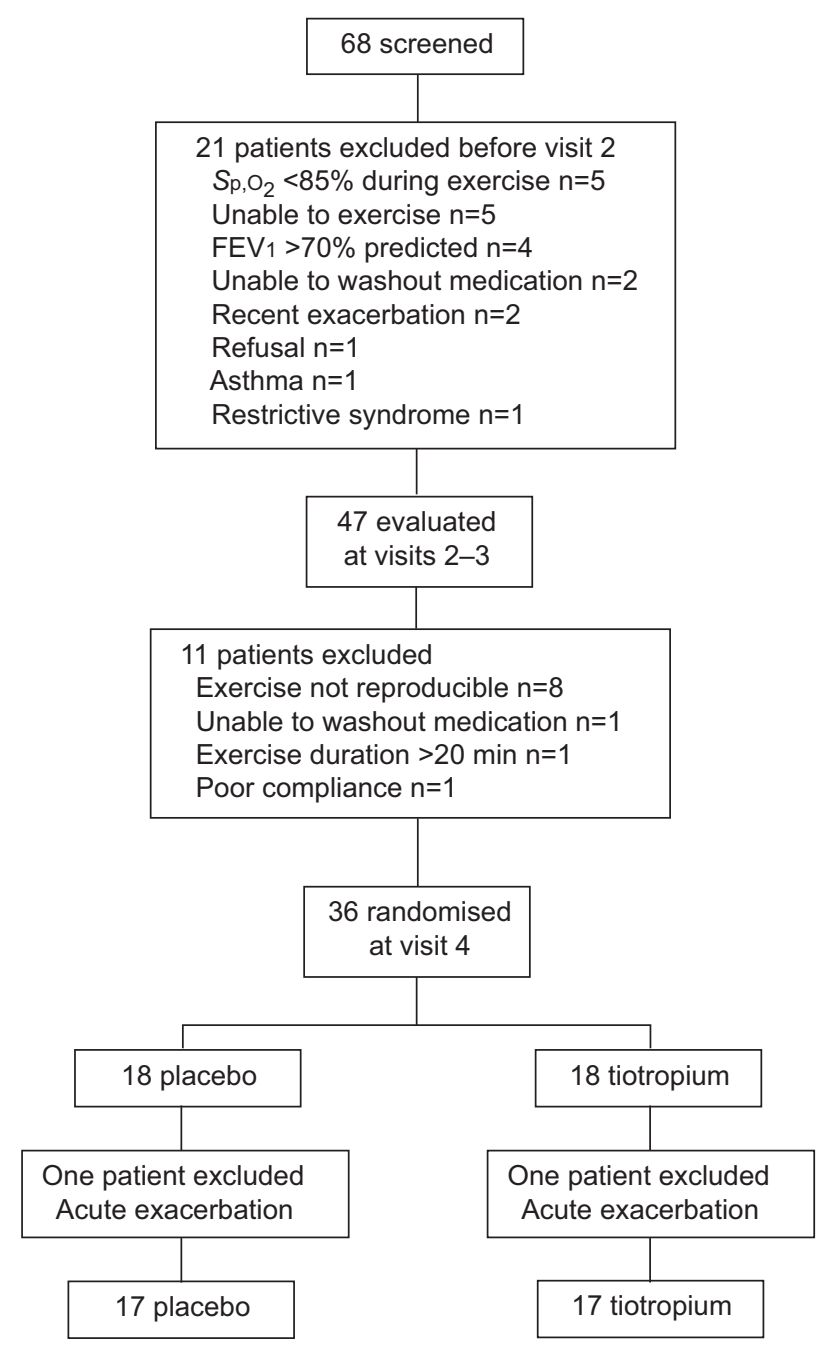

FIGURE 1. Study flow chart. $\mathrm{Sp}_{\mathrm{p}} \mathrm{O}_{2}$ : arterial oxygen saturation measured by pulse oximetry; FEV1: forced expiratory volume in $1 \mathrm{~s}$.

comparison with the control group, the two groups were well matched for baseline characteristics.

\section{Endurance time and walking distance}

Group mean changes in walking endurance time after the first study dose of medication and after 3 weeks of treatment are shown in figure $2 \mathrm{a}$, while the individual changes in walking endurance time at 3 weeks are provided in figure $2 b$. After the first dose, there was no significant change in walking endurance time compared with baseline in either the tiotropium group $(38 \pm 142 \mathrm{~s})$ or the placebo group $(-29 \pm 124 \mathrm{~s})$. After 3 weeks of treatment, tiotropium was associated with a significant improvement in walking endurance time compared with baseline $(132 \pm 166 \mathrm{~s} ; \mathrm{p}<0.001)$ with no change in the placebo groups $(4 \pm 111 \mathrm{~s})$. The between-group difference (tiotropium-placebo) amounted to $128 \pm 141(\mathrm{p}=0.017) .12(71 \%)$ patients out of 17 in the active treatment group improved their walking endurance time beyond the minimal important difference for this outcome parameter (65s), while this magnitude of improvement was seen in only four $(24 \%)$ out of 17 patients in the placebo group $(\mathrm{p}=0.006)$. 


\begin{tabular}{|c|c|c|}
\hline & Placebo & Tiotropium \\
\hline Subjects $n$ & 17 & 17 \\
\hline Males & $11(65)$ & $12(71)$ \\
\hline Age yrs & $66 \pm 8$ & $64 \pm 7$ \\
\hline Body mass index $\mathrm{kg} \cdot \mathrm{m}^{-2}$ & $26.0 \pm 3.0$ & $29.4 \pm 4.4$ \\
\hline FEV 1 L & $1.42 \pm 0.47$ & $1.53 \pm 0.51$ \\
\hline FEV $1 \%$ pred & $54 \pm 12$ & $53 \pm 12$ \\
\hline FVC \% pred & $102 \pm 18$ & $99 \pm 15$ \\
\hline FEV $_{1} /$ FVC $\%$ & $41 \pm 10$ & $44 \pm 11$ \\
\hline FRC $\%$ pred $^{\#}$ & $138 \pm 22$ & $132 \pm 31$ \\
\hline TLC $\%$ pred $^{\#}$ & $116 \pm 12$ & $111 \pm 15$ \\
\hline RV \% pred ${ }^{\#}$ & $148 \pm 28$ & $138 \pm 37$ \\
\hline IC \% pred & $91 \pm 15$ & $86 \pm 17$ \\
\hline DL,Co \% pred & $57 \pm 13$ & $56 \pm 19$ \\
\hline Peak $\mathrm{Vo}_{2} \mathrm{~mL} \cdot \mathrm{kg}^{-1} \cdot \mathrm{min}^{-1}$ & $17.1 \pm 4.4$ & $16.9 \pm 4.0$ \\
\hline Peak V'E L. $\min ^{-1}$ & $47.2 \pm 17.5$ & $52.0 \pm 16.0$ \\
\hline Peak V'E/MVV \% & $92 \pm 12$ & $94 \pm 14$ \\
\hline Peak $S_{p}, O_{2} \%$ & $92 \pm 3$ & $91 \pm 3$ \\
\hline Peak heart rate beats $\mathrm{min}^{-1}$ & $127 \pm 14$ & $126 \pm 19$ \\
\hline Endurance time $s$ & $391 \pm 81$ & $404 \pm 114$ \\
\hline \multicolumn{3}{|l|}{ Respiratory medication } \\
\hline Short-acting $\beta_{2}$-agonists & $12(71)$ & $13(76)$ \\
\hline Long-acting $\beta_{2}$-agonists & $3(18)$ & $3(18)$ \\
\hline Short-acting anticholinergics & $1(6)$ & $1(6)$ \\
\hline Long-acting anticholinergics & $6(35)$ & $6(35)$ \\
\hline Inhaled corticosteroids & $2(12)$ & $1(6)$ \\
\hline $\begin{array}{l}\text { Long-acting } \beta_{2} \text {-agonists/inhaled } \\
\text { corticosteroids }\end{array}$ & $7(41)$ & $12(71)$ \\
\hline
\end{tabular}

Data are presented as $\mathrm{n}(\%)$ or mean $\pm \mathrm{SD}$, unless otherwise indicated. FEV1: forced expiratory volume in $1 \mathrm{~s}$; \% pred: \% predicted; FVC: forced vital capacity; FRC: functional residual capacity; TLC: total lung capacity; RV: residual volume; IC: inspiratory capacity; $D \mathrm{~L}, \mathrm{CO}$ : single-breath diffusing capacity of the lung for carbon monoxide; $\mathrm{VO}_{2}$ : oxygen uptake; $V^{\prime} \mathrm{E}$ : minute ventilation; $\mathrm{MVV}$ : maximum voluntary ventilation; $\mathrm{Sp}, \mathrm{O}_{2}$ : arterial oxygen saturation measured by pulse oximetry. ${ }^{*}: \mathrm{n}=16$.

\section{Pulmonary function testing}

The comparative effects of tiotropium and placebo on pulmonary function are shown in figure 3. At 3 weeks, the trough values for FEV1 and FVC were significantly improved with tiotropium in comparison with placebo (tiotropium-placebo): $80 \pm 111 \mathrm{~mL}$ $(p=0.05)$ and $174 \pm 264 \mathrm{~mL}(\mathrm{p}=0.043)$, respectively. The reduction in trough functional residual capacity (FRC), residual volume (RV) and total lung capacity tended to be larger with tiotropium compared with placebo, but the between-group comparison did not reach statistical significance. The post-dose response to tiotropium was statistically superior to placebo after the first dose of study medication and after 3 weeks of treatment. The between-group differences at 3 weeks amounted to: FEV1 $(129 \pm 131 \mathrm{~mL} ; \mathrm{p}=0.002), \mathrm{FVC}(295 \pm 292 \mathrm{~mL} ; \mathrm{p}=0.008)$, inspiratory capacity $(151 \pm 217 \mathrm{~mL} ; \mathrm{p}=0.03)$ and $\mathrm{RV}(-287 \pm 500 \mathrm{~mL}$; $\mathrm{p}=0.06$ ). The post-dose reduction in FRC at 3 weeks with tiotropium compared with placebo was numerically important $(-199 \pm 547 \mathrm{~mL})$, but this difference did not reach statistical significance. The changes in total lung capacity throughout the study were small and not statistically significant.

\section{Physiological response during ESWT}

Changes in physiological response to exercise after 3 weeks of treatment (visit 5) in comparison with baseline values in both study groups are reported in table 2 . In general, the changes were small and not statistically significant. Ventilation and tidal volume at the end of the ESWT were significantly improved at 3 weeks in the tiotropium group in comparison with placebo. The Borg dyspnoea score did not change significantly in either group at isotime or peak exercise. Symptoms responsible for exercise limitation were not modified; at baseline, $14(83 \%)$ patients of the tiotropium group and $13(76 \%)$ patients in the placebo group stopped because of dyspnoea. After 3 weeks of treatment, 14 $(83 \%)$ patients in each group stopped because of dyspnoea.

\section{DISCUSSION}

This study provides an additional confirmation that the ESWT enables the detection of functional changes after bronchodilation in patients with COPD. Tiotropium, a long-acting anticholinergic
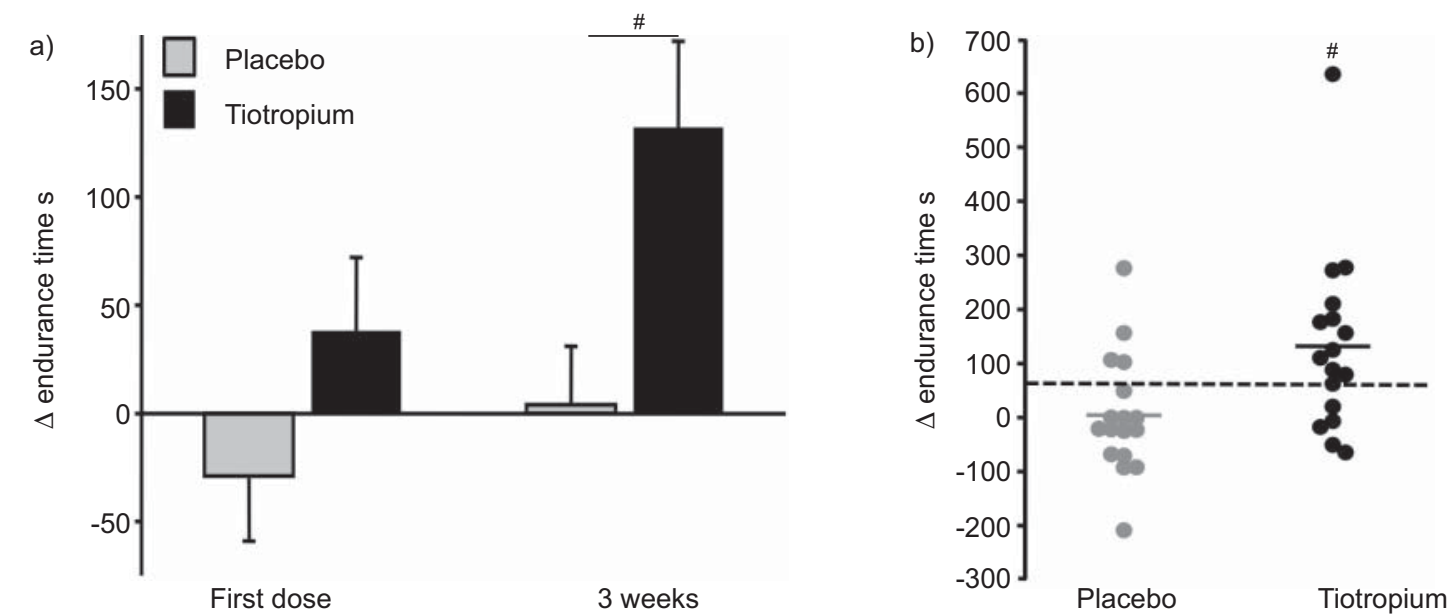

FIGURE 2. a) Group mean \pm SEM changes in walking endurance time after the first dose and after 3 weeks of treatment compared with baseline value and b) individual changes in walking endurance time at 3 weeks. The grey and black horizontal lines in b represent the group mean change seen in the placebo and tiotropium groups, respectively, while the dashed line represents the minimal important difference for the endurance shuttle walk test (65 s). The between-group difference in the improvement in walking endurance time was statistically significant at 3 weeks, whereas it was not different after a single dose $(p=0.12)$. ${ }^{*}$ : between-group comparison $p=0.017$. 
a)

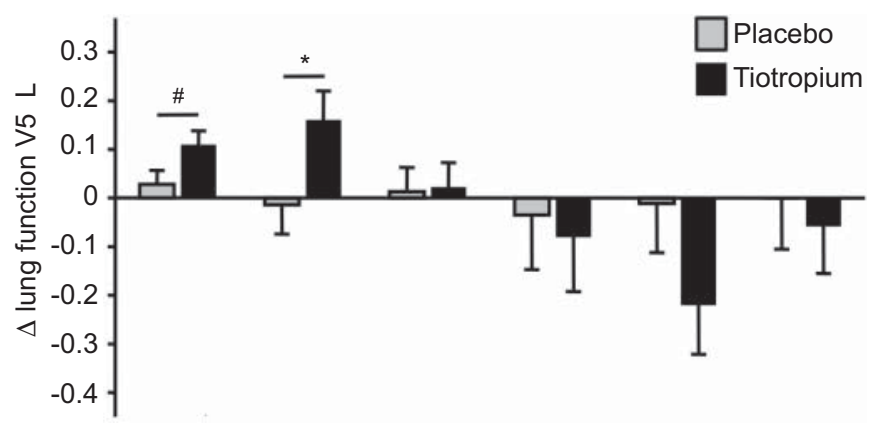

b)

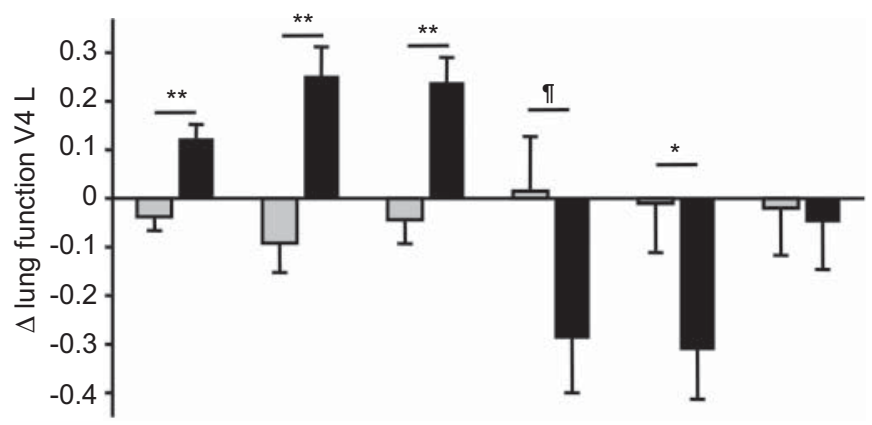

C)

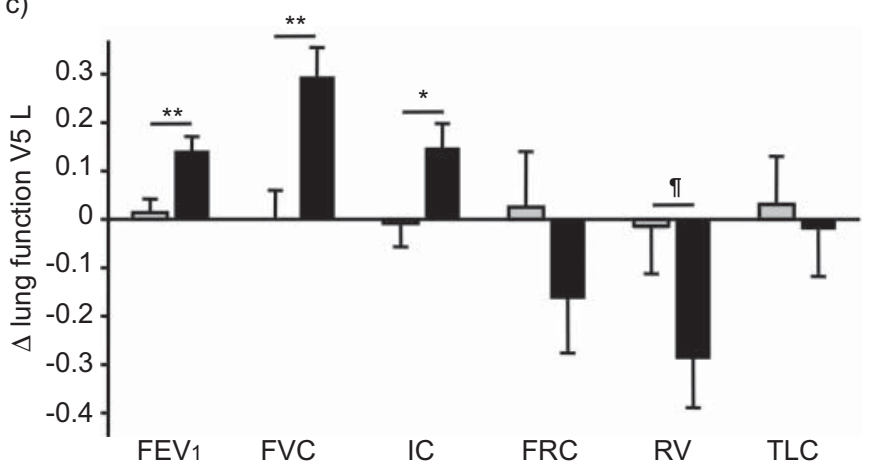

FIGURE 3. Mean \pm SEM changes in resting pulmonary function. The trough responses at a) 3 weeks (visit (V)5) in comparison with baseline, and the post-study medication responses b) after the first dose (V4) and c) after 3 weeks (V5) in comparison with baseline are represented. FEV1: forced expiratory volume in $1 \mathrm{~s}$; FVC: forced vital capacity; IC: inspiratory capacity; FRC: functional residual capacity; $\mathrm{RV}$ : residual volume; TLC: total lung capacity. ${ }^{*}: p=0.05 ;{ }^{\bullet}: p=0.06 .{ }^{*}: p<0.05$; $\star *: p<0.01$.

bronchodilator, significantly improved walking capacity in patients with moderate-to-severe COPD after 3 weeks of treatment. It also significantly increased expiratory flows and inspiratory capacity while promoting lung deflation. This study provides the first confirmation that tiotropium improves walking capacity beyond the minimal important difference for this outcome in patients with COPD.

The ESWT is a simple field exercise test that was initially developed to assess exercise capacity in patients with COPD [15]. More recently, we assessed the evaluative properties of this exercise testing modality [6-8]. We demonstrated that the ESWT was more sensitive than the constant-load cycling exercise test in detecting an improvement in functional status following

\begin{tabular}{|c|c|c|c|}
\hline \multirow[t]{2}{*}{ TABLE 2} & \multicolumn{3}{|c|}{$\begin{array}{l}\text { Changes in physiological responses to exercise } \\
\text { at } 3 \text { weeks compared with baseline }\end{array}$} \\
\hline & Placebo & Tiotropium & p-value \\
\hline Subjects n & 17 & 17 & \\
\hline \multicolumn{4}{|l|}{$V^{\prime} E L \cdot \min ^{-1}$} \\
\hline Rest & $-0.2 \pm 4.1$ & $-0.6 \pm 5.2$ & 0.783 \\
\hline Isotime & $-0.6 \pm 2.6$ & $1.18 \pm 5.4$ & 0.240 \\
\hline Peak & $-1.2 \pm 2.5$ & $2.9 \pm 4.2$ & 0.002 \\
\hline \multicolumn{4}{|c|}{$f R$ breaths $\cdot \mathrm{min}^{-1}$} \\
\hline Rest & $1.1 \pm 4.8$ & $-2.3 \pm 5.5$ & 0.064 \\
\hline Isotime & $0.8 \pm 2.9$ & $-0.8 \pm 5.2$ & 0.283 \\
\hline Peak & $-0.3 \pm 2.6$ & $0.7 \pm 3.4$ & 0.319 \\
\hline \multicolumn{4}{|l|}{ VT $\mathrm{mL}$} \\
\hline Rest & $-70 \pm 210$ & $0 \pm 320$ & 0.228 \\
\hline Isotime & $-40 \pm 120$ & $80 \pm 200$ & 0.032 \\
\hline Peak & $-30 \pm 100$ & $60 \pm 130$ & 0.026 \\
\hline \multicolumn{4}{|l|}{$V^{\prime} O_{2} L \cdot \min ^{-1}$} \\
\hline Rest & $0.01 \pm 0.11$ & $0.00 \pm 0.12$ & 0.851 \\
\hline Isotime & $-0.02 \pm 0.07$ & $0.00 \pm 0.11$ & 0.503 \\
\hline Peak & $-0.02 \pm 0.07$ & $0.02 \pm 0.11$ & 0.258 \\
\hline \multicolumn{4}{|c|}{$V^{\prime} \mathrm{CO}_{2} \mathrm{~L} \cdot \mathrm{min}^{-1}$} \\
\hline Rest & $0.00 \pm 0.09$ & $0.04 \pm 0.18$ & 0.372 \\
\hline Isotime & $-0.05 \pm 0.08$ & $0.00 \pm 0.15$ & 0.288 \\
\hline Peak & $-0.08 \pm 0.12$ & $0.04 \pm 015$ & 0.023 \\
\hline \multicolumn{4}{|c|}{ Dyspnoea Borg scale } \\
\hline Rest & $0.2 \pm 0.7$ & $-0.2 \pm 0.7$ & 0.162 \\
\hline Isotime & $-0.5 \pm 2.0$ & $-0.9 \pm 1.7$ & 0.586 \\
\hline Peak & $-0.1 \pm 0.7$ & $0.1 \pm 1.3$ & 0.521 \\
\hline \multicolumn{4}{|l|}{$\mathrm{Sp}, \mathrm{O}_{2}$} \\
\hline Rest & $0 \pm 2$ & $1 \pm 1$ & 0.252 \\
\hline Isotime & $1 \pm 2$ & $2 \pm 2$ & 0.281 \\
\hline Peak & $1 \pm 2$ & $1 \pm 2$ & 0.923 \\
\hline \multicolumn{4}{|l|}{ fc } \\
\hline Rest & $-3 \pm 13$ & $-6 \pm 7$ & 0.516 \\
\hline Isotime & $-4 \pm 10$ & $-3 \pm 7$ & 0.886 \\
\hline Peak & $-2 \pm 17$ & $-1 \pm 8$ & 0.751 \\
\hline
\end{tabular}

Data are presented as mean \pm SD, unless otherwise stated. Isotime was defined as the latest exercise time that was reached both at baseline and at 3 weeks during the endurance shuttle walk test. $V^{\prime} E$ : minute ventilation; fR: respiratory rate; VT: tidal volume; $V^{\prime} \mathrm{O}_{2}$ : oxygen uptake; $V^{\prime} \mathrm{CO}_{2}$ : carbon dioxide production; $\mathrm{Sp}_{1} \mathrm{O}_{2}$ : arterial oxygen saturation measured by pulse oximetry; fc: cardiac

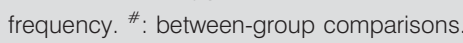

bronchodilation in patients with COPD [6]. This observation that walking is a well-suited exercise modality to detect improvement in functional status following bronchodilation was further corroborated by other investigators [19]. One likely mechanism for this observation is that walking, as opposed to cycling, induces significantly less quadriceps fatigue [6, 20], which is a phenomenon that prevents bronchodilation to fully translate into better exercise capacity [21, 22]. The relative magnitude of improvement in walking capacity with tiotropium was larger with the ESWT (32\%) than that reported for the ISWT $(11 \%)$ [23]. This is in keeping with the notion that constant loadbased exercise protocols are more responsive than incremental maximum tests [24]. 
Another attractive feature of the ESWT is that walking is an activity relevant to daily living. Previous studies have documented the ability of tiotropium to improve the 6-min walking distance [25] and the incremental shuttle walking distance [23], but the magnitude of these improvements was small and of unclear clinical significance [26, 27]. In contrast, the 128-s improvement in walking endurance after 3 weeks of tiotropium in comparison with placebo is clinically important, as it was recently suggested that a mean 65-s (95\% CI 45-85 s) change in the ESWT endurance time is likely to be perceived by patients [17]. In this context, the present study provides clinically relevant information about the impact of tiotropium on walking capacity. We also noted that the improvement in walking capacity was small after the first dose of tiotropium. This conforms with the pharmacology of this medication and with previous studies showing that the exercise-enhancing effects of tiotropium are modest after a single dose and increase progressively over a 3-6week period [1, 3, 23, 25].

The effects of tiotropium on lung function seen in the present study were generally consistent with the expected improvement in expiratory flows reported for this bronchodilator. However, the magnitude of lung deflation was somewhat smaller than in two studies in which patients were selected on the basis of a FRC $\geqslant 120 \%$ pred $[1,3]$ and in which the mean FRC was $160-170 \%$ pred. In the present study, baseline hyperinflation was not a prerequisite for participation and the mean FRC was 135\% pred; as such, the possibility of observing pronounced lung deflation was likely to be reduced.

Monitoring the physiological responses during walking using a portable metabolic system makes it possible to explore the mechanisms of improvement in walking capacity with bronchodilation. In this study, after 3 weeks of treatment with tiotropium, we observed a significant increase in ventilation and in tidal volume at end-exercise of the ESWT. These improvements were likely to be associated with an improved maximum voluntary ventilatory capacity, a consequence of both bronchodilation with improved maximal expiratory flows and increased inspiratory capacity due to reduced air trapping [28]. A fundamental benefit of bronchodilation is the reduction of operating lung volumes during exercise [28, 29]. Although we did not measure inspiratory capacity during exercise, the improved inspiratory capacity at rest prior to exercise indicates that patients were breathing at lower lung volumes at the beginning of exercise, an advantage expected to be maintained throughout the exercise duration [28]. In addition, the expansion in tidal volume at isotime and end-exercise is likely to be a reflection of a reduced mechanical constraint and operating lung volumes during exercise [28]. This effect on tidal volume is important given its association with improved exercise tolerance [28].

Some methodological comments should be made about our study to help in its interpretation. Although our experience with the ESWT in assessing the response to bronchodilation is favourable [6-8], these studies were conducted in a single centre and the feasibility of this exercise testing modality in a multicentre setting remains to be confirmed, as was previously done for the endurance cycling exercise test $[1,3,5,30]$. The measurement of inspiratory capacity is also more challenging during a walking test in which the patient is not stationary [7]. This may be viewed as a potential limitation of a walking test as an outcome measure in an exercise study for which a thorough physiological assessment is considered an important objective. The study sample size was too small to fully explore the sensory responses during exercise. A reduction in isotime dyspnoea would be an expected consequence of the improved ventilatory mechanics that were observed with tiotropium, but this could not be confirmed in the present study. However, the similar dyspnoea score at endexercise between the two groups, in spite of the increased endurance time with tiotropium, may suggest that the rate of increase in dyspnoea during walking was reduced following treatment with tiotropium. A larger clinical trial will be useful to address the impact of tiotropium on dyspnoea during walking. We did not perform a true intent-to-treat analysis, as two patients who presented an exacerbation of their disease were excluded from the analysis, as pre-specified by the study protocol. This analysis strategy is in keeping with studies evaluating the effects of bronchodilation on exercise tolerance in COPD [3,5]. Although the sample size was adequate to provide a clear answer to the research question, it was not sufficiently large to explore the exercise-enhancing effects of tiotropium in various subgroups of patients with COPD. We acknowledge the short-term duration of our study. Considering that tiotropium maintains its bronchodilatory properties over time [31], we suspect that the exerciseenhancing effects of this drug should also persist in the longterm. However, a study properly designed to evaluate the longterm impact of tiotropium on walking capacity in COPD will be necessary to address this issue.

In conclusion, the present study demonstrates the ability of tiotropium to significantly improve walking capacity in patients with COPD after 3 weeks of treatment. Reduction of airway obstruction leading to lung deflation and enhanced maximal ventilation and end-exercise tidal volume contribute to this enhanced exercise capacity. This study supports the role of the ESWT in evaluating the functional gain and physiological impact of interventions in patients with COPD.

\section{SUPPORT STATEMENT}

This work was supported by an unrestricted educational grant from Boehringer Ingelheim and Pfizer (Canada). The sponsor (Boehringer Ingelheim and Pfizer) was not involved in the study design, data collection, analysis or interpretation. The sponsor had the opportunity to read and comment on the manuscript with no obligation for the authors to incorporate any suggestion into the final version. This study is registered at Clinical Trials. gov identifier number NCT01307189.

\section{STATEMENT OF INTEREST}

Statements of interest for V. Pepin and F. Maltais and for this study can be found at www.erj.ersjournals.com/site/misc/statements.xhtml

\section{ACKNOWLEDGEMENTS}

The authors acknowledge the help of M. Bélanger, M-J. Breton, B. Jean and J. Picard (all at Institut Universitaire de Cardiologie et de Pneumologie de Québec, Québec, Canada) in performing the study. They also thank E. Nadreau for technical support, and G. Daigle and S. Simard (Université Laval, Québec) for statistical assistance.

\section{REFERENCES}

1 O'Donnell DE, Flüge T, Gerken F, et al. Effects of tiotropium on lung hyperinflation, dyspnoea and exercise tolerance in COPD. Eur Respir J 2004; 23: 832-840. 
2 O'Donnell DE, Voduc N, Fitzpatrick M, et al. Effect of salmeterol on the ventilatory response to exercise in chronic obstructive pulmonary disease. Eur Respir J 2004; 24: 86-94.

3 Maltais F, Hamilton A, Marciniuck D, et al. Improvements in symptom-limited exercise performance over eight hours with once-daily tiotropium in patients with COPD. Chest 2005; 128 $1168-1178$.

4 Neder JA, Fuld JP, Overend T, et al. Effects of formoterol on exercise tolerance in severely disabled patients with COPD. Respir Med 2007; 101: 2056-2064.

5 Maltais F, Celli B, Casaburi R, et al. Aclidinium bromide improves exercise endurance and lung hyperinflation in patients with moderate to severe COPD. Respir Med 2011; 105: 580-587.

6 Pepin V, Saey D, Whittom F, et al. Walking versus cycling: sensitivity to bronchodilation in chronic obstructive pulmonary disease. Am J Respir Crit Care Med 2005; 172: 1517-1522.

7 Brouillard C, Pepin V, Milot J, et al. Endurance shuttle walking test: responsiveness to salmeterol in COPD. Eur Respir J 2008; 31: 579-584.

8 Pepin V, Brodeur J, Lacasse Y, et al. Six-minute walking versus shuttle walking: responsiveness to bronchodilation in chronic obstructive pulmonary disease. Thorax 2007; 63: 291-298.

9 O'Donnell DE, Travers J, Webb KA, et al. Reliability of ventilatory parameters during cycle ergometry in multicentre trials in COPD. Eur Respir J 2009; 34: 866-874.

10 Miller MR, Hankinson J, Brusasco V, et al. Standardisation of spirometry. Eur Respir J 2005; 26: 319-338.

11 Pellegrino R, Viegi G, Brusasco V, et al. Interpretative strategies for lung function tests. Eur Respir J 2005; 26: 948-968.

12 Macintyre N, Crapo RO, Viegi G, et al. Standardisation of the single-breath determination of carbon monoxide uptake in the lung. Eur Respir J 2005; 26: 720-735.

13 Quanjer PH, Tammeling GJ, Cotes JE, et al. Lung volumes and forced ventilatory flows. Report working party standardization of lung function tests, european community for steel and coal. Official statement of the European Respiratory Society. Eur Respir J 1993; 6: Suppl. 16, 5-40.

14 Singh SJ, Morgan MD, Scott S, et al. Development of a shuttle walking test of disability in patients with chronic airways obstruction. Thorax 1992; 47: 1019-1024.

15 Revill SM, Morgan MD, Singh SJ, et al. The endurance shuttle walk: a new field test for the assessment of endurance capacity in chronic obstructive pulmonary disease. Thorax 1999; 54: 213-222.

16 Guyatt GH, Pugsley S, Sullivan MJ, et al. Effect of encouragement on walking test performance. Thorax 1984; 39: 818-822.

17 Pepin V, Laviolette L, Brouillard C, et al. Significance of changes in endurance shuttle walking performance. Thorax 2011; 66: 115-120.
18 Borg G. Psychophysical bases of perceived exertion. Med Sci Sports Exer 1982; 14: 377-381.

19 Zhang X, Waterman LA, Ward J, et al. Advantages of endurance treadmill walking compared with cycling to assess bronchodilator therapy. Chest 2010; 137: 1354-1361.

20 Man WD, Soliman MG, Gearing J, et al. Symptoms and quadriceps fatigability after walking and cycling in chronic obstructive pulmonary disease. Am J Respir Crit Care Med 2003; 168: 562-567.

21 Saey D, Debigaré R, LeBlanc $\mathrm{P}$, et al. Contractile leg fatigue after cycle exercise: a factor limiting exercise in patients with COPD. Am J Respir Crit Care Med 2003; 168: 425-430.

22 Deschênes D, Pepin V, Saey D, et al. Locus of symptom limitation and exercise response to bronchodilation in chronic obstructive pulmonary disease. J Cardiopulm Rehabil Prev 2008; 28: 208-214.

23 Verkindre C, Bart F, Aguilaniu B, et al. The effect of tiotropium on hyperinflation and exercise capacity in chronic obstructive pulmonary disease. Respiration 2006; 73: 420-427.

24 Oga T, Nishimura K, Tsukino M, et al. The effects of oxitropium bromide on exercise performance in patients with stable chronic obstructive pulmonary disease. A comparison of three different exercise tests. Am J Respir Crit Care Med 2000; 161: 1897-1901.

25 Okudan N, Gok M, Gokbel H, et al. Single dose of tiotropium improves the 6-minute walk distance in chronic obstructive pulmonary disease. Lung 2006; 184: 201-204.

26 Redelmeier DA, Bayoumi AM, Goldstein RS, et al. Interpreting small differences in functional status: the six-minute walk test in chronic lung disease patients. Am J Respir Crit Care Med 1997; 155: 1278-1282.

27 Singh SJ, Jones P, Evans R, et al. The minimum clinically important improvement for the incremental shuttle walking test. Thorax 2008; 63: 775-777.

28 O'Donnell DE, Lam M, Webb KA. Spirometric correlates of improvement in exercise performance after anticholinergic therapy in chronic obstructive pulmonary disease. Am J Respir Crit Care Med 1999; 160: 542-549.

29 Marin JM, Carrizo SJ, Gascon M, et al. Inspiratory capacity, dynamic hyperinflation, breathlessness, and exercise performance during the 6-minute-walk test in chronic obstructive pulmonary disease. Am J Respir Crit Care Med 2001; 163: 1395-1399.

30 O'Donnell DE, Sciurba F, Celli B, et al. Effect of fluticasone propionate/salmeterol on lung hyperinflation and exercise endurance in COPD. Chest 2006; 130: 647-656.

31 Tashkin DP, Celli B, Senn S, et al. A 4-year trial of tiotropium in chronic obstructive pulmonary disease. N Engl J Med 2008; 359: 1543-1554. 